\title{
Nanodielectrics with giant permittivity
}

\author{
S K SAHA \\ Department of Materials Science, Indian Association for the Cultivation of Science, Kolkata 700 032, India
}

\begin{abstract}
Nanodielectrics is an emerging area of research because of its potential application in energy storage and transducers. One-dimensional metallic nanostructures with localized electronic wave functions show giant dielectric constant. Following the prediction, during the last couple of years we have investigated the effect of giant permittivity in one-dimensional systems of conventional metals and conjugated polymer chains. In this article, we have tried to summarize the works on giant permittivity and finally the fabrication of nanocapacitor using metal nanowires, which shows giant permittivity is also discussed.
\end{abstract}

Keywords. Nanodielectrics; giant permittivity; electronic wave functions.

\section{Introduction}

Nanodielectrics, is the subject of study related to dielectric phenomena of nanoscopic materials having morphology of particles, sheets, wires and tubes etc (Lewis 1994). During the last two decades, numerous research results have been published in different areas of nanotechnology such as carbon nanotubes, nanowires, nanodiodes, single electron transistors, molecular data storage and drug delivery etc, but surprisingly, the area of nanodielectrics is relatively unexplored, although, there is enormous scope to use this material in power electronics industry and as gate electrodes in thin film transistors. For example, to reduce the operating voltage of organic thin film transistor is a great challenge these days. By using high- $k$ dielectric as gate electrode, this operating voltage has been reduced significantly in organic thin film transistor and even in silicon technology, the high- $k$ gate material is increasingly being used for potential advantages. Therefore, it is worth to summarize the results so far published in the area of nanodielectrics with a brief outline for the future application in nanodevices.

The work on nanodielectrics started a couple of years back and most of them are comprised of composite materials in which nano sized particles are embedded in insulating matrices (Alamdari 2000; Chang et al 2000; Mukherjee and Chakravorty 2002; Utracki 2003). The dielectric performance of these composite materials is primarily controlled by the interface (which is a nanophase) between the particles and the matrix. In particular, it is shown that the enhanced area of interface changes the bonding state, which affects the interfacial polarization. But the main limitation of the composite materials is its use in nanodevices. Therefore, few efforts have been

(cnssks@iacs.res.in) directed to explore the possibility of using the intrinsic dielectric property of nano materials in electronic devices. A thin film $(\sim 8 \mathrm{~nm})$ of high- $k$ zirconium oxide has been used as gate material in single walled carbon nanotube field effect transistor (FET) to push the performance limit for molecular electronics (Javey et al 2002). Very recently, ultra thin $(2 \cdot 3-5.5 \mathrm{~nm})$ self-assembled organic dielectric multilayers have been integrated into organic thin film transistors to achieve sub-volt operating characteristics (Yoon et al 2005).

Although, some studies on dielectric composites (Roy and Chakravorty 1993; Kundu and Chakravorty 1995) and organic dielectrics have been carried out, the field of nanodielectrics with giant permittivity value remains in its infant stage. In 1965, Gor'kov and Eliashberg predicted an enormously high permittivity value of a sufficiently minute metal particle having discrete energy levels (Gor'kov and Eliashberg 1965a, b). Later, this result was modified with interrupted metal strand instead of particle due to depolarization field (Chen et al 2001). In the last decade few works have been done in composite materials in which high permittivity value has been reported. It is only recently reported (Gor'kov and Eliashberg 1965a, b) by the present author (Saha 2004), a giant $\left(10^{10}\right)$ permittivity value in an assembly of ultra fine silver metal particles as a result of quantum confinement of the electronic wave functions. These materials with extremely high dielectric permittivity will be useful as ultracapacitors for energy storage if the loss factor is controlled tuning the disorder for electron confinement. Therefore, it is not difficult to speculate that an emerging area of nanodielectrics with a new class of materials having ultra high permittivity is imminent to be used in the future application in energy storage and ultra sensitive transducers. The objective of the present article is to assemble works on nanodielectrics with giant permittivity value, which have been investigated during the last couple of years by the present author. 


\subsection{Background of experiments}

In 1972, Strassler et al pointed out the intriguing possibility that there might be a class of metallic systems for which the effect of depolarization field would be negligible. They interpreted this anomalous result of high dielectric constant considering 'interrupted strand model' - a real crystalline compound consisting of a system of collinear metallic strands, each of which is interrupted by a series of perfectly insulating lattice defects. A small distribution of such defects replaces the defect free strand by a linear sequence of 1D metallic box.

According to this strand model the expression for this dielectric constant was calculated as

$$
\varepsilon \sim\left(q_{\mathrm{s}} l_{0}\right)^{2}
$$

where $q_{\mathrm{s}}$ is the Fermi-Thomas screening wave vector of the conduction electrons and $l_{0}$ the strand length.

\section{Experimental}

\subsection{Synthesis of silver nanowires}

Silver nanowires have been grown in the particle tracketched polycarbonate membrane with pore diameter, $15 \mathrm{~nm}$, by electro-deposition technique using silver nitrate as the electrolyte. For electro-deposition, one face of the membrane was coated by silver paste (supplied by $\mathrm{M} / \mathrm{s}$ Acheson Colloiden B. V. Holland), which acted as the cathode. The freshly synthesized samples were washed several times by double distilled water to remove all unreacted silver nitrate. The membrane containing the nanowires was then dried in vacuum oven and the other electrode was fabricated by the same silver paste.

\subsection{Synthesis of gold nanowires}

Gold nanowires have been grown inside a porous anodic alumina template with pore diameter, $\sim 60 \mathrm{~nm}$, by electro-deposition technique. In the first step, porous anodic alumina (PAA) membrane was formed by a two-step anodization process using aluminum foil. In the second step, gold nanowires were electrodeposited into the pores of PAA under galvanostatic conditions using a commercial solution (Orotemp 24, Technic Inc).

\subsection{Synthesis of polypyrrole nanotubes}

The conducting polypyrrole nanorods were synthesized by our own technique reported earlier. Anodic alumina membrane with pore density, $10^{8} / \mathrm{cm}^{2}$ and pore diameter, $100 \mathrm{~nm}$, was immersed in ammonium per sulphate, $\left(\mathrm{NH}_{4}\right)_{2} \mathrm{~S}_{2} \mathrm{O}_{8}$. Due to capillary action, ammonium per sulphate solution entered into the channels of the membrane.
The membrane was then dried at $70 \mathrm{C}$ for $3 \mathrm{~h}$ in a vacuum oven. The dried membrane was then dipped in aqueous pyrrole solution for $12 \mathrm{~h}$ at room temperature for complete polymerization. Finally all samples were washed several times by distilled water to remove all un-reacted ammonium per sulphate.

\subsection{Nanodielectric sample preparation}

In the first step gold microelectrodes and probing pads for electrical measurements were made on a Si wafer by photolithography (Saha 2006), Au deposition and photoresist lift off techniques. In the second step, the gold nanowires were separated from the membrane by dissolving it into $\mathrm{NaOH}$ and after successive centrifugation and sensing with deionized (DI) water. The nanowires were then manipulated in between two microelectrodes by dielectrophoresis technique (Rice and Bernasconi 1972; Saha 2006). For this the substrate containing the microelectrodes was then dipped into the solution of nanowires and using an a.c. signal with frequency, $1 \mathrm{MHz}$ and bias, $15 \mathrm{~V}$. The nanowires were aligned in between the microelectrodes due to electrostatic force.

For microstructural study of the nanowires, nanotubes and device, we have used TEM (Model, JEM 2011 made

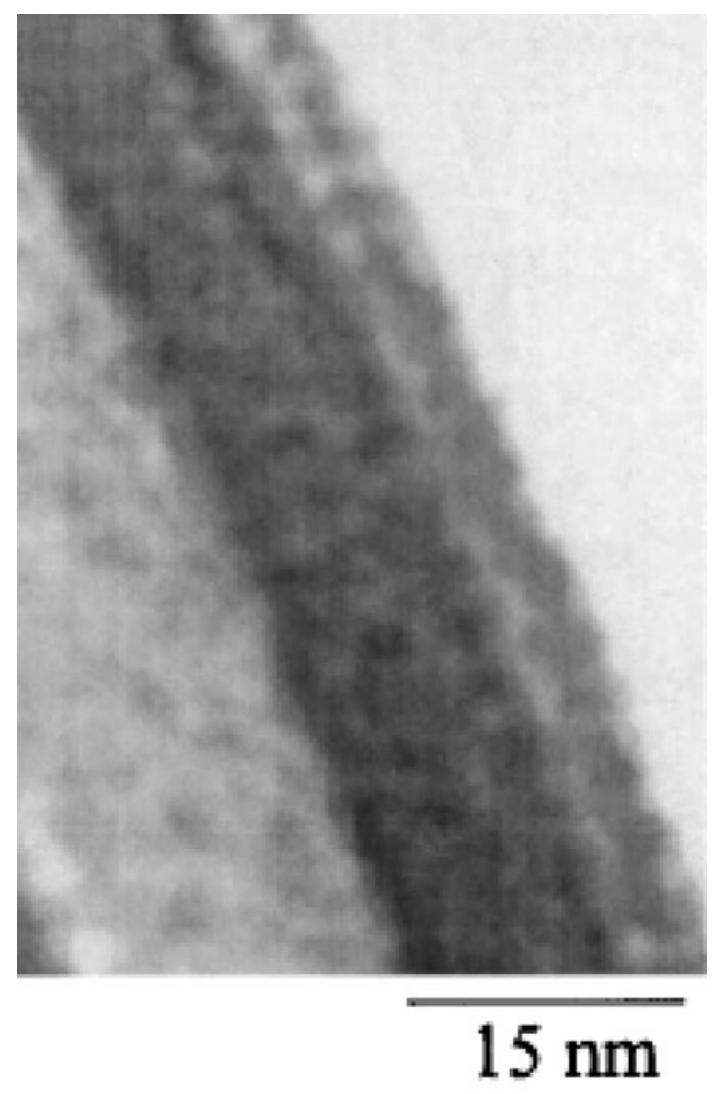

Figure 1. TEM micrograph of the silver nanowire, $15 \mathrm{~nm}$ in diameter. 
by JEOL) and FE-SEM (Model JSM 6700F) and for dielectric measurements we have used HP 4284A LCR Meter and for current-voltage measurements HP 4155 B Semiconductor Parameter Analyser was used.

\section{Results and discussion}

\subsection{Ag nanowires}

It was shown earlier (Fleury 1997) that nanowires formed by electro-deposition technique, is composed of ultra fine particles the size of which depends on the growth rate. Figure 1 shows the TEM micrograph of silver nanowires formed by particles with size, $\sim 1 \mathrm{~nm}$. When a voltage pulse of $5 \mathrm{~V}$ is applied across the nanowire, it goes into the molten state with the formation of an assembly of ultra fine particles due to the high current density $\left(10^{8} \mathrm{~A} / \mathrm{m}^{2}\right)$. Under the application of electric field these particles will be isolated due to the local melting in the neck regions between the particles with the enhancement of resistance from $1.2 \mathrm{ohm}$ to $2.5 \mathrm{Mohm}$. Such ultra fine isolated particles are shown in figure 2 . The capacitance value was measured at $1 \mathrm{MHz}$ from which the dielectric constant was calculated to be $\sim 4$. It means that the isolated particles

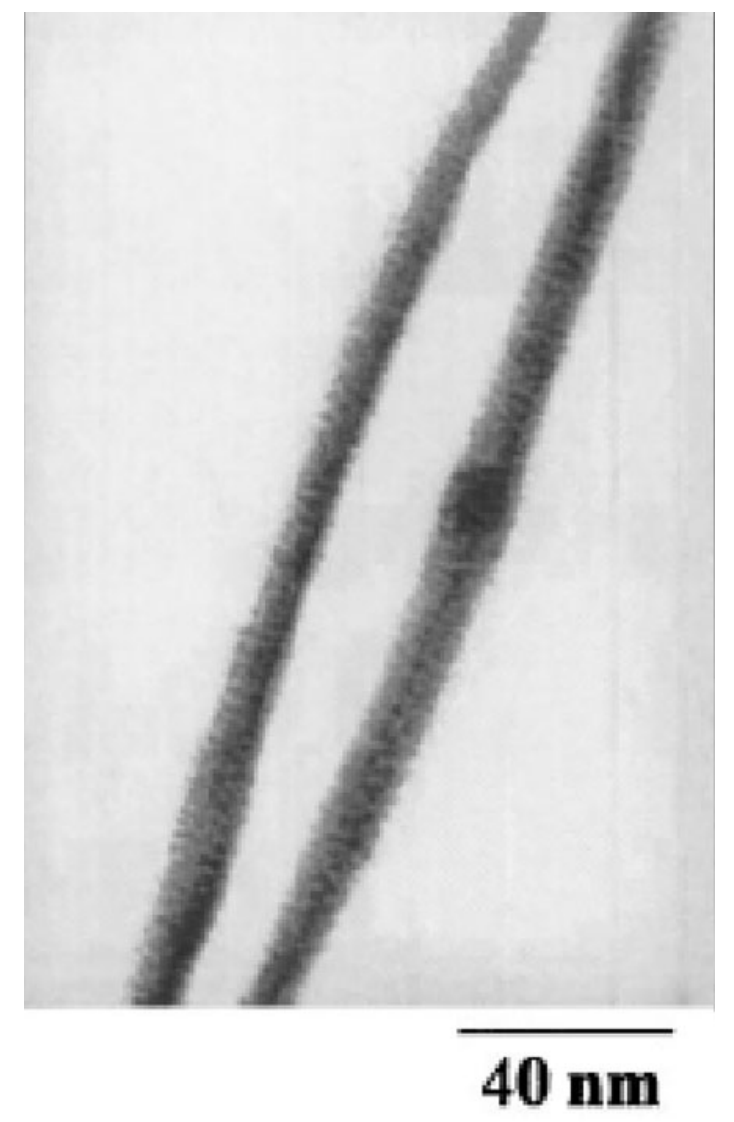

Figure 2. TEM micrograph of silver wire after voltage application showing the assembly of isolated ultra fine particles forming the wire do not show any enhancement of dielectric constant as a result of Gor'kov-Eliashberg effect. This is because of the fact that particles formed due to melting are spherical in shape and due to high depolarization field of the spherical particles the enhancement of the 'effect' was not observed.

But under the application of electric field the capacitance increases very sharply with voltage and then becomes saturated as shown in figure 3 . The corresponding resistance behaviour, which decreases with voltage up to a certain value and becomes saturated, is also shown. This is because of the fact that the spherical particles are elongated in the direction of the applied electric field due to strong electrostatic force acting in the nanogap between the particles to form a metal filament. This metal

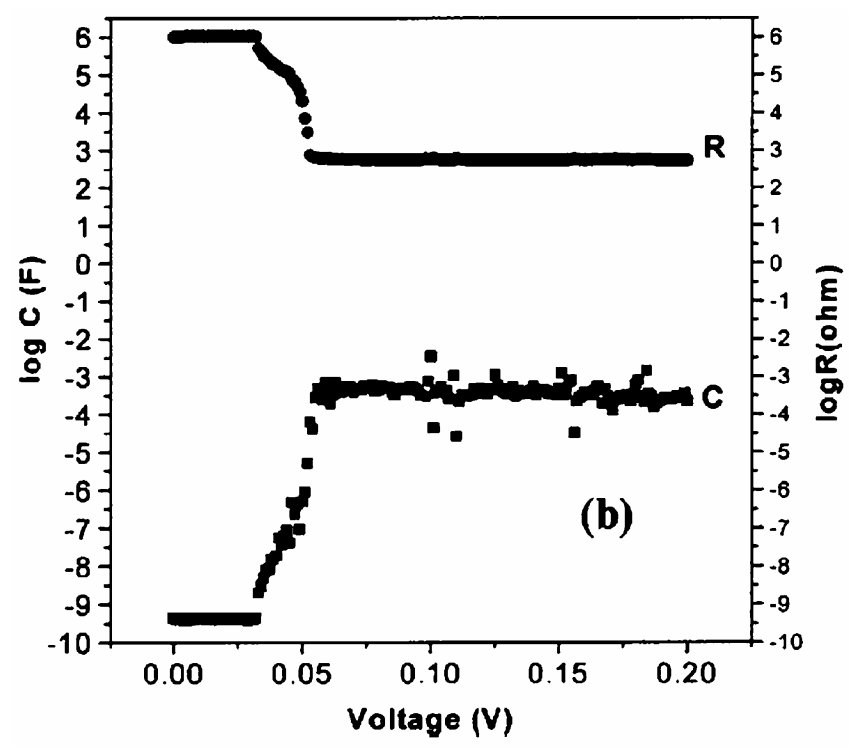

Figure 3. Variation of capacitance and resistance with voltage at $1 \mathrm{kHz}$.

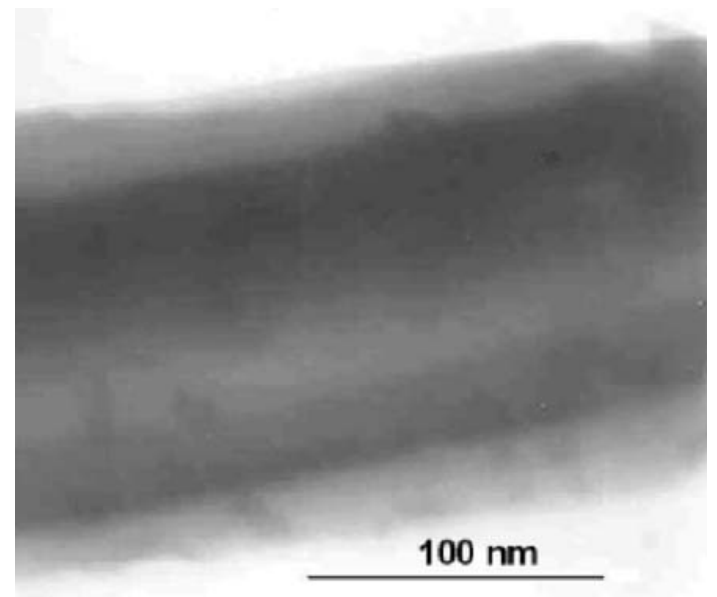

Figure 4. TEM micrograph of the polypyrrole nanorod. 
filament can be considered as a metal strand the electronic wave functions of which are localized due to lattice defects at the interface. Therefore, strand model has been applied to calculate the dielectric permittivity. Taking a length of the wire as $50 \mu \mathrm{m}$ the permittivity has been calculated as $\varepsilon \sim 10^{10}$ by the formula given in (1). A value of $q_{\mathrm{s}}^{2} \sim 1 \times 10^{19} / \mathrm{m}^{2}$ has been used for this calculation. The same permittivity value has also been calculated using the measured capacitance and the effective sample dimension by the formula $C t / \varepsilon_{0} \cdot A$, where $t$ is the thickness, $A$ the area and $\varepsilon_{0}$ the free space dielectric permittivity.

\subsection{Polypyrrole nanorods}

Template guided polypyrrole nanorods have been synthesized by chemical route. Figure 4 shows the TEM micrograph of the nanorods. In conjugated polymers, the polymer chains are metallic in nature but chemical defects, crosslinking and entanglement of the chains cause to behave

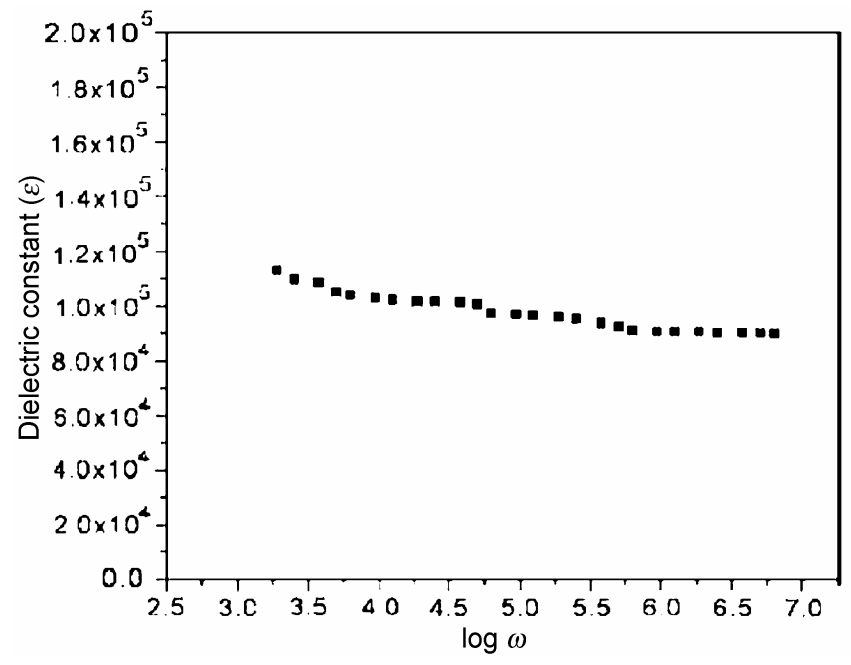

Figure 5. Variation of dielectric constant with frequency of the polypyrrole nanorod.
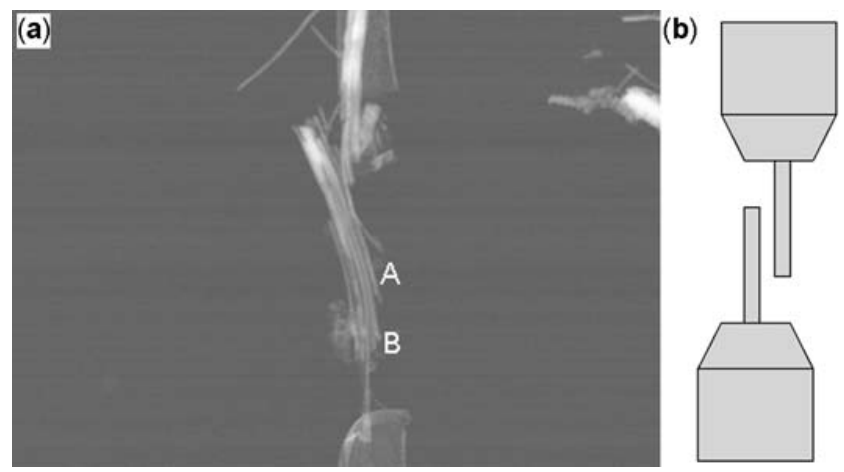

Figure 6. (a) SEM micrograph of the nanocapacitor fabricated using gold nanowires and (b) schematic diagram of the nanocapacitor. like a semiconductor in their bulk phase. In the earlier work (Bhattacharyya and Saha 2002), it is seen that nanotubes with wall thickness, $10 \mathrm{~nm}$, show metallic behaviour with a positive temperature coefficient of resistance. The origin of this metallic behaviour is the structural ordering and chain alignment along the length of the nanotube. Therefore, the polymer nanotubes can be considered as a large number of aligned chains interconnected to each other. The aligned chains form an ordered phase and the interconnected portions form a disordered phase (Saha 2002). In the ordered phase the electronic wave functions are in the extended state and in the disordered phase the wave functions are in the localized state. The localization depends on the extent of disorder. For solid rod like structure (diameter, $100 \mathrm{~nm}$ ), the growth is almost three-dimensional and the interconnected portions are more disordered. Therefore, the aligned chains in the solid rod-like structure

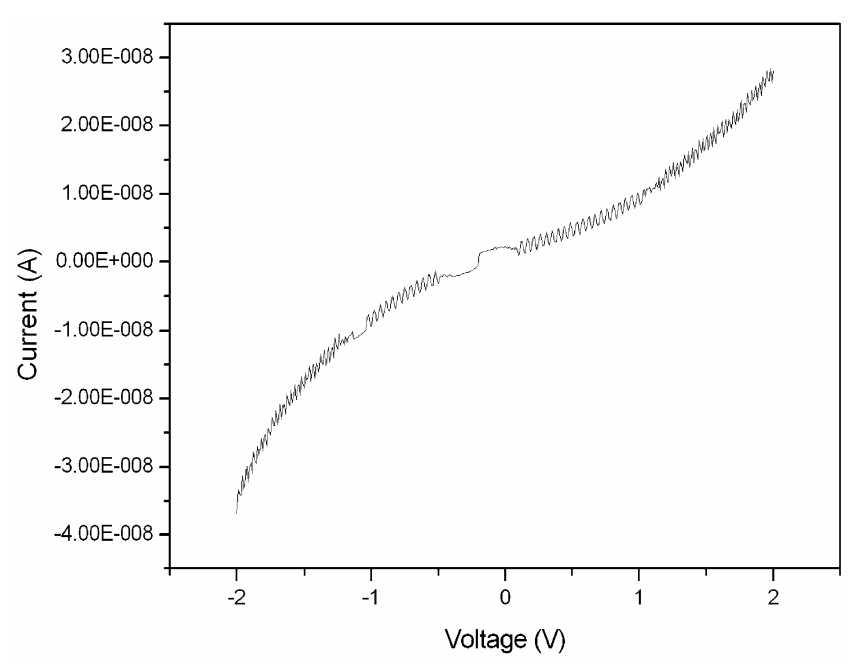

Figure 7. Current-voltage characteristics of the nanocapacitor.

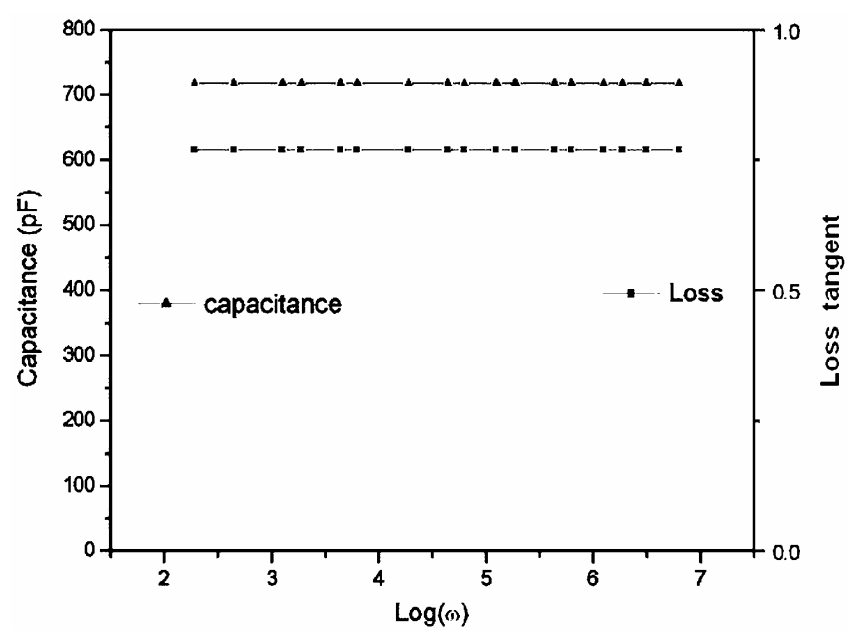

Figure 8. Variation of capacitance and loss factor of the nanocapacitor with frequency. 
are the ideal systems to consider as the disordered metal filaments to study G-E effect (Saha and Chakravorty 2006). The dielectric permittivity has been calculated from the measured capacitance value of these nanorods to be $\varepsilon \sim 10^{5}$ as shown in figure 5 . Using this value in (1), the chain length (strand length) is estimated to be $l_{0} \sim 100 \mathrm{~nm}$.

\subsection{Application}

Electro-deposited metal nanowires have been used to fabricate nanocapacitor. In the specific sample structure (Saha et al 2006), gold nanowires (serving as nanoelectrodes) have been manipulated in between two microelectrodes by dielectrophoresis technique. A schematic of the capacitor is also shown in figure 6. It is common in all nanostructures that the surface atoms are loosely bounded compared to the core atoms. During dielectrophoresis a voltage of $15 \mathrm{~V}$ was used to manipulate the nanowires. When nanowires from the two microelectrodes come very close to each other as shown in figure 6 there is a very high electric field acting between the wires. Therefore, the surface gold atoms may come out and get trapped at the interface as a result the interface between the nanoelectrodes form a disordered phase with isolated gold atoms.

Figure 7 gives the current-voltage behaviour of the device from which it is seen that the current is nonlinear and fluctuating up to room temperature. This fluctuation arises due to nano contacts of the gold atoms or particles with the nanowires. The variation of capacitance and the corresponding loss factor with frequency is shown in figure 8 . From the figure it is seen that the capacitance and loss factor remain constant over the frequency range $30 \mathrm{~Hz}$ to $1 \mathrm{MHz}$, which is due to wide distribution of relaxation times. Considering a parallel plate capacitor the dielectric permittivity has been calculated using the measured capacitance to be $\sim 10^{7}$. This high value of permittivity at the interface between the wires has been attributed to the presence of gold atoms which increase the density of states at the interface.

\section{Conclusions}

It is seen that one-dimensional nanostructures of metal filaments or conducting polymer chain segments with localized electronic wave functions show giant dielectric permittivity. By using novel properties of ultra fine metal particles, it is possible to fabricate nanodielectrics with giant permittivity values between two metal nanoelectrodes to form a nanocapacitor with ultra high capacitance. The loss factor can be controlled by tailoring the structures at the nanoscale level or attaching some insulating units at the two ends of the filament.

\section{Acknowledgement}

This work was done under special grant of Nano Initiative (NSTI), DST, New Delhi, Govt. of India.

\section{References}

Alamdari H D 2000 Ph D Thesis, Laval University, Quebec, Canada

Bhattacharyya S and Saha S K 2002 Appl. Phys. Lett. 804612

Chang S, Doremus R H, Ajayan P M and Siegel R W 2000 Ceram. Eng. Sci. Proc. 21653

Chen X Q, Saito T, Yamada H and Matsushige K 2001 Appl. Phys. Lett. 783714

Fleury V 1997 Nature (London) 390145

Gor'kov L P and Eliashberg G M 1965a Zh. Eksp. Teor. Fiz. 48 1407

Gor'kov L P and Eliashberg G M 1965b Sov. Phys. JETP 21940

Javey A, Kim H, Brink M, Wang Q, Ural A, Guo J, Mcintyre P, Mceuen P, Lundstrom M and Dai H 2002 Nature Mater. 1 241

Krupke R, Hennrich F, Lohneysen H and Kappers M 2003 Science 301344

Kundu T K and Chakravorty D 1995 Appl. Phys. Lett. 672732

Lewis T J 1994 IEEE Trans. Dielectr. Electr. Insul. 1812

Mukherjee P K and Chakravorty D 2002 J. Mater. Res. 173127

Rice M J and Bernasconi J 1972 Phys. Rev. Lett. 29113

Roy B and Chakravorty D 1993 J. Mater. Res. 1206

Saha S K 2002 Appl. Phys. Lett. 813645

Saha S K 2004 Phys. Rev. B69 125416

Saha S K and Chakravorty D 2006 Appl. Phys. Lett. 89043117

Saha S K, Dasilva M, Hang Qingling, Sands T and Janes D B 2006 Nanotechnology 172284

Strassler S, Rice M J and Wider P 1972 Phys. Rev. B6 2575

Utracki L A 2003 Proc. 2nd int. symp. polymer nanocomposites science technology (Boucherville, Quebec, Canada: NRCIMI)

Yoon M-H, Facchetti A and Marks T J 2005 PNAS 1024678 